\title{
Haemoglobin and ferritin concentrations in infants at 8 months of age
}

\author{
A M Emond, N Hawkins, C Pennock, Jean Golding and the ALSPAC Children in Focus \\ Team
}

\begin{abstract}
Aim-To identify the optimum age to screen for iron deficiency, the normal distribution of haemoglobin and ferritin in a representative population sample was investigated.

Methods-Normal values for haemoglobin and ferritin were measured from heel prick capillary samples obtained from a representative cohort of 1175 infants at 8 months old who were randomly selected from children taking part in the Avon Longitudinal Study of Pregnancy and Childhood (ALSPAC).

Results-Haemoglobin was normally distributed: mean (SD) 117 (11) g/1, 95\% confidence interval (CI) 116 to 118 , and range 72-153 g/l. Ferritin was $\log$ normally distributed: geometric mean $38.5 \mu \mathrm{g} / 1,95 \%$ CI $37 \cdot 0$ to $39 \cdot 9$, range $7 \cdot 1-224 \mu$ g/l. The 5 th centile for haemoglobin was $97 \mathrm{~g} / \mathrm{l}$ and for ferritin $16.9 \mu \mathrm{g} / \mathrm{l}$. No correlation was found between haemoglobin and ferritin.

Multiple regression analysis showed ferritin concentrations to be positively related to birth weight $(p<0.0001)$ and the sex of the child (girls with higher concentrations) $(p<0.0001)$ but negatively with the child's weight at 8 months $(p<0 \cdot 0001)$. Haemoglobin concentrations were positively related to the child's weight at 8 months $(p=0 \cdot 04)$. Neither haemoglobin nor ferritin concentrations were related to social class as measured by maternal education level.
\end{abstract}

Conclusion-These data define the normal range for haemoglobin and ferritin in capillary samples in the UK population, and suggest that anaemia is common in infancy. Using current recommendations, $23 \%$ of infants would be identified as anaemic. For British infants at 8 months of age, a more representative 'cut off' for anaemia would be haemoglobin concentration $<97 \mathrm{~g} / \mathbf{l}$ and for iron deficiency ferritin $<\mathbf{1 6} \mu \mathrm{g} / \mathbf{l}$.

(Arch Dis Child 1996; 74: 36-39)

Keywords: haemoglobin, ferritin, iron deficiency.

Institute of Child

Health, Royal Hospital for Sick Children, St

Michael's Hill, Bristol

BS2 8BJ

A M Emond

N Hawkins

C Pennock

J Golding

Correspondence to: Dr Emond.

Accepted 11 August 1995

Iron deficiency is thought to be a common problem in young children in inner city populations, ${ }^{1}$ with associated effects on growth and development. $^{2}$ The definition of anaemia which is commonly used, $<110 \mathrm{~g} / \mathrm{l}$, is recommended by the World Health Organisation (WHO), ${ }^{3}$ but the relevance of this cut off point to UK populations is not known. Many of the published data on normal haemoglobin and the definition of anaemia are derived from studies in the developing world 4 with few contemporary population based data from the UK. ${ }^{5-8}$

In order to identify the optimum age to screen for iron deficiency we are investigating the normal distribution of haemoglobin and ferritin and assessing the relationship of these to the developmental progress of the child, in a representative population sample from the Avon Longitudinal Study of Pregnancy and Childhood (ALSPAC). This is a geographically based cohort study investigating factors influencing health and development of children, and provides an ideal medium for determining the current distributions of haemoglobin and ferritin concentrations in young children.

\section{Methods}

All births from the Bristol and District Health Authority area with an expected date of delivery between 1 April 1991 and 31 December 1992 were eligible. Over $80 \%$ of the known births from the geographically defined catchment area were included, resulting in a total cohort of 14185 surviving live births. From this population cohort, a randomly selected sample of 1560 mothers (whose babies were born within the last six months of survey) were invited to bring their 8 month old children to a research clinic (Children in Focus), where a number of clinical, physiological, and psychological assessments were carried out.

A heel prick sample of capillary blood was taken from the children and collected into an EDTA bottle. The haemoglobin concentration was assayed using the HEMOCUE B-haemoglobin photometer. The blood was then centrifuged and the plasma removed and frozen. Plasma ferritin was assayed using the DELFIA time resolved fluoroimmunoassay system. If the samples had clotted they were discarded. Sufficient blood was obtained to measure haemoglobin concentration for 1075 children and sufficient plasma was obtained to assay ferritin for 754 children. All blood samples were processed within four days of sampling.

The volume of blood obtained was small and therefore it was not possible to perform all assays in duplicate. For those where a double assay was feasible, coefficients of variation were calculated. The haemoglobin and ferritin assays had coefficients of variation of approximately $3 \%$ and $4 \%$ respectively. The assays used in this study were calibrated using supplied standards. In addition they were 


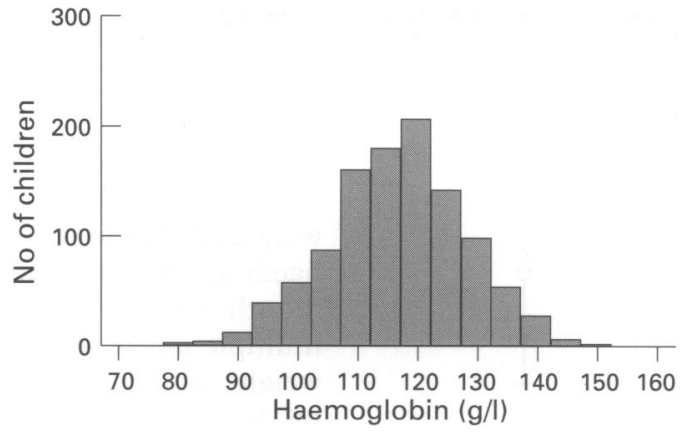

Figure 1 Haemoglobin distribution at 8 months of age.

compared with those used in the local haematology department at the Bristol Royal Infirmary. This laboratory utilises the national external quality assurance scheme. Assays were compared for ferritin $(n=24)$ and haemoglobin $(n=19)$. The median ratio of the results of the ferritin DELFIA assay to the Bristol Royal Infirmary assay was 0.85 (range $0.69-1.29$ ), implying that the DELFIA assay indicated lower ferritin concentrations than the clinical assay. The median difference between our HEMOCUE system and the Bristol Royal Infirmary assays was $+3 \mathrm{~g} / \mathrm{l}$ (range 0 to $+7 \mathrm{~g} / \mathrm{l}$ ), indicating that the HEMOCUE assay gave a slightly higher haemoglobin concentration.

In order to test the stability of the samples three separate exercises were undertaken. First, five blood samples were assayed both on day 0 (day when sample was obtained) and on day 13; the mean difference in haemoglobin was $1 \%$, ranging from $-1 \%$ to $+4 \%$. Second, five plasma samples were assayed on day 0 and day 6; the mean difference in plasma ferritin was $+5 \%$, ranging from $-5 \%$ to $+13 \%$. Third, five plasma samples were assayed on day 0 and frozen at $-20^{\circ} \mathrm{C}$. After thawing they were reassayed; the mean difference in plasma ferritin was $0 \%$, ranging from $-5 \%$ to $+4 \%$.

Ethical approval of the study was obtained from the ALSPAC ethics committee, and the local ethics committees of United Bristol, Southmead and Frenchay Healthcare Trusts. Following their advice, the results of those children whose haemoglobin was found to be less than $90 \mathrm{~g} / \mathrm{l}$ were given to the mothers, with a letter to hand to their general practitioner suggesting further investigation.

\section{Results}

Of the 1560 mothers invited to the clinic 1318 $(85 \%)$ attended. The majority $(95 \%)$ of the

Table 1 Haemoglobin centiles $(g / l)$

\begin{tabular}{lccr}
\hline Centile & All children & Boys & Girls \\
\hline 5th & 97 & 96 & 99 \\
10th & 102 & 101 & 103 \\
20th & 108 & 107 & 109 \\
30th & 112 & 111 & 112 \\
40th & 114 & 114 & 115 \\
50th & 117 & 117 & 118 \\
60th & 120 & 120 & 120 \\
70th & 123 & 123 & 123 \\
80th & 126 & 126 & 127 \\
90th & 131 & 132 & 131 \\
95th & 136 & 136 & 135 \\
No of children & 1075 & 595 & 480 \\
\hline
\end{tabular}

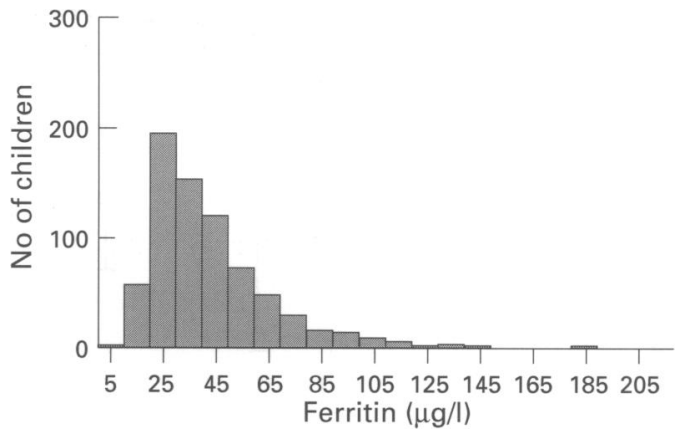

Figure 2 Ferritin distribution at 8 months of age.

children were between 8 and 8.5 months of age. Of the mothers who attended the clinic, $1161(88 \%)$ gave their permission for blood samples to be taken from their 1175 children. There were 1147 singleton and 28 twin children; 652 boys and 523 girls. Only $2 \%$ of mothers were non-white.

\section{HAEMOGLOBIN CONCENTRATIONS}

The haemoglobin concentrations ranged from 72 to $153 \mathrm{~g} /$ (fig 1 ). The arithmetic mean (SD) haemoglobin concentration at 8 months of age was $117(11) \mathrm{g} / \mathrm{l}(\mathrm{n}=1074)$ with $95 \%$ confidence interval (CI) 116 to $118 \mathrm{~g} / \mathrm{l}$. The mean haemoglobin for boys $(n=595)$ was $117 \mathrm{~g} / \mathrm{l}(95 \% \mathrm{CI}$ 116 to 118 ). This was not statistically significantly different from that for girls $(n=480): 117$ g/l (95\% CI 116 to 118$)$. The centiles, calculated from ranked data, are shown in table 1.

\section{FERRITIN CONCENTRATIONS}

Sufficient plasma was available to measure plasma ferritin concentrations for 754 children. Plasma ferritin was found to be log normally distributed and ranged from $7 \cdot 2$ to $223.6 \mu \mathrm{g} / \mathrm{l}$. The geometric mean was $38.5 \mu \mathrm{g} / 1$ ( $95 \%$ CI 37.0 to $39.9 \mu \mathrm{g} / \mathrm{l})$. The distribution is illustrated in fig 2 , and the centiles, based on rank order, are shown in table 2.

The mean plasma ferritin for boys $(n=418)$ was $35.5 \mu \mathrm{g} / \mathrm{l}(95 \% \mathrm{CI} 33.8$ to 37.4$)$, and that for girls $(\mathrm{n}=336)$ was $42 \cdot 4 \mu \mathrm{g} / 1 \mathrm{l}(95 \%$ CI $40 \cdot 1$ to $44 \cdot 8)$. This difference was statistically significant $(\mathbf{p}<0 \cdot 001, t$ test $)$.

CURRENT GUIDELINES

Using the recommended WHO definition of anaemia (haemoglobin $<110 \mathrm{~g} / \mathrm{l}$ ),${ }^{3} 23 \%$ of the surveyed children from Avon would be

Table 2 Ferritin centiles $(\mu g /)$

\begin{tabular}{lccc}
\hline Centile & All children & Boys & Girls \\
\hline 5th & $16 \cdot 9$ & $15 \cdot 2$ & $20 \cdot 2$ \\
10th & $20 \cdot 8$ & $18 \cdot 7$ & $23 \cdot 1$ \\
20th & $25 \cdot 0$ & $23 \cdot 5$ & $27 \cdot 1$ \\
30th & $28 \cdot 3$ & $26 \cdot 5$ & $31 \cdot 2$ \\
40th & $32 \cdot 3$ & $29 \cdot 9$ & $35 \cdot 1$ \\
50th & $37 \cdot 7$ & $35 \cdot 2$ & $41 \cdot 3$ \\
60th & $42 \cdot 8$ & $39 \cdot 9$ & $47 \cdot 1$ \\
70th & $49 \cdot 5$ & $45 \cdot 5$ & $56 \cdot 0$ \\
80th & $58 \cdot 6$ & $53 \cdot 3$ & $64 \cdot 3$ \\
90th & $75 \cdot 7$ & $69 \cdot 1$ & $85 \cdot 7$ \\
95th & $98 \cdot 8$ & $89 \cdot 5$ & $105 \cdot 6$ \\
No of children & 754 & 418 & 336 \\
\hline
\end{tabular}




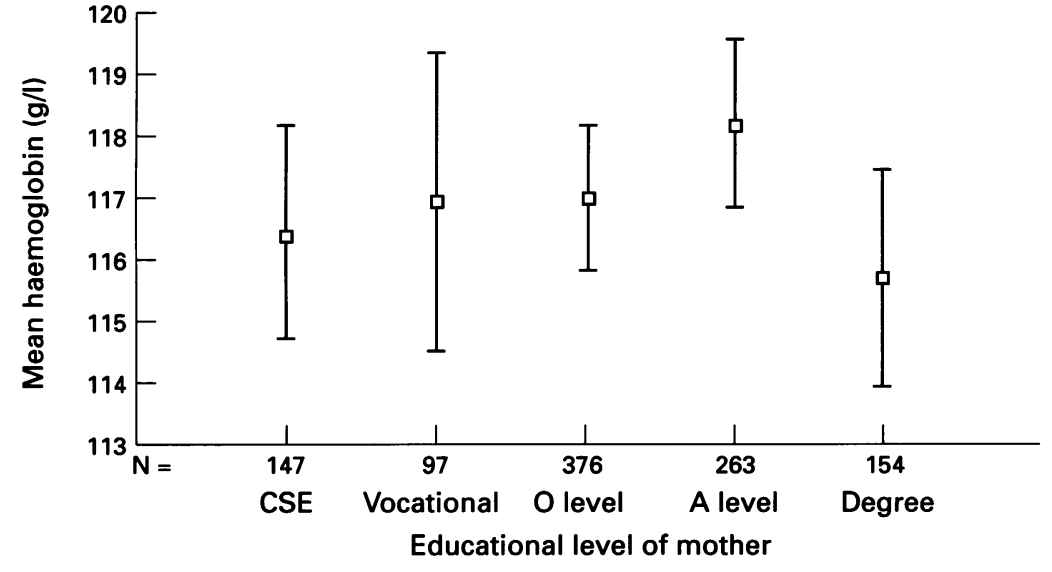

Figure 3 Relationship between children's haemoglobin concentrations and maternal education; bars show 95\% CI of the mean. (CSE group includes those with no formal qualifications; $A$ level includes those with nursing and teaching qualifications.)

regarded as anaemic. The cut off recommended by the American NHANES II study ${ }^{9}$ of $107 \mathrm{~g} / 1$ would identify $20 \%$ of this cohort as anaemic. Using a plasma ferritin concentration of $<12 \mu \mathrm{g} / \mathrm{l}$ as a definition of iron deficiency, $1 \cdot 2 \%$ of this population cohort of Avon children fell below this line. Only $0.4 \%$ of the population fell below both cut offs.

\section{UNADJUSTED ASSOCIATIONS}

There was no significant correlation between haemoglobin and log ferritin (Pearson's correlation coefficient $r=0.0440, \mathrm{p}=0.231, \mathrm{n}=742$ ) nor between haemoglobin and sex or haemoglobin and birth weight. There was, however, a significant positive association between haemoglobin and the weight of the child at 8 months $(r=0.063, \mathrm{p}=0.040, \mathrm{n}=1072)$.

There were significant positive associations between log ferritin and birth weight (Pearson's correlation coefficient $r=0.248$, $\mathrm{p}<0.001, \mathrm{n}=754$ ) but a significant negative association between 8 month clinic weight and $\log$ ferritin (Pearson's correlation coefficient $r=-0 \cdot 101, \mathrm{p}=0.006, \mathrm{n}=751$ ).

There were no statistically significant differences between maternal education level and either the children's haemoglobin $(p=0.260$, analysis of variance (ANOVA), $F=1.323$, $n=1037)$ or ferritin ( $p=0.835$, ANOVA,

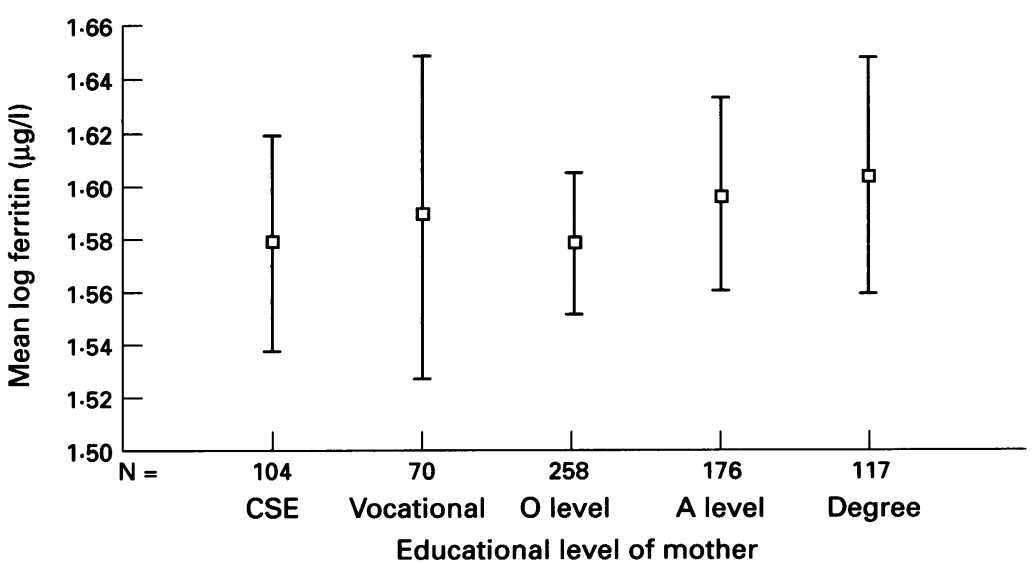

Figure 4 Relationship between children's ferritin concentrations and maternal education; bars show 95\% CI of the mean. (CSE group includes those with no formal qualifications; $A$ level includes those with nursing and teaching qualifications.)
$\mathrm{F}=0 \cdot 363, \mathrm{n}=725$ ) concentrations (figs 3 and 4).

\section{ADJUSTED ASSOCIATIONS}

The independent associations of sex, birth weight, and 8 month clinic weight in predicting haemoglobin and ferritin concentrations at 8 months of age were investigated using stepwise multiple linear regression. The 8 month clinic weight was the only factor that entered the model relating to haemoglobin concentration, and only explained $0.4 \%$ of the variance (table 3). For ferritin, however, all three items entered the model and were able to explain $13 \%$ of the variance (table 4); girls had higher concentrations than boys, the heavier the birth weight but the lower the current weight, the higher the plasma ferritin concentration.

\section{Discussion}

This study, based on a representative unselected sample, is the largest population based study measuring haemoglobin and ferritin in British children. The population of Avon is very similar to the UK national average, and the Children in Focus study group has been derived from a mixed urban and rural population and from all social classes. These data therefore represent the best and most accurate current assessment of the normal range for these measurements in white infants of this age.

Our data challenge the assumption that social circumstances are related to reduced iron stores or anaemia. We have shown no relationship between either haemoglobin or ferritin concentrations and social class, nor with ethnicity (although numbers of minority groups were small) at this age. The negative association between ferritin and weight at 8 months suggest that the more rapid the growth of the child, the more depleted the iron stores, but the positive relationship between weight and haemoglobin is not easy to explain.

In common with other studies, we have shown that haemoglobin concentrations are normally distributed. We found a mean at 8 months of $117 \mathrm{~g} / \mathrm{l}$. This age is traditionally felt to reflect the physiological nadir of haemoglobin ${ }^{9}$ but in fact there have been few population based studies of such young children. Interestingly there was a population study in part of the ALSPAC study area in $1966 . .^{5}$ Capillary blood samples taken at 9 months were reported to have a mean (SD) haemoglobin of 116 (11) $\mathrm{g} / 1$, almost identical to these results obtained 27 years later.

It should be stressed that these data are derived from capillary blood samples, which are recognised to produce lower haemoglobin concentrations than equivalent venous samples ${ }^{10}$; ferritin concentrations are, however, comparable. ${ }^{11}$ Generally, the normal range of laboratory values (test reference) is based on the test results of the central $95 \%$ distribution of 'healthy' individuals. We have used a population sample (reference population) to define our normal range, without 
Table 3 Results of stepwise linear regression on haemoglobin concentration $(g / l)$

\begin{tabular}{lllll}
\hline Variable & Adjusted regression coefficient & $95 \%$ CI & t & $p$ Value \\
\hline Weight at 8 months $(\mathrm{kg})$ & $0.68^{\star}$ & 0.03 to 1.33 & 2.2 & 0.0305
\end{tabular}

$R^{2}=0.004 ; \mathrm{n}=1071$

${ }^{\star}$ Equivalent to an increase in haemoglobin of $0.68 \mathrm{~g} / 1$ for a $1 \mathrm{~kg}$ increase in weight.

Table 4 Results of stepwise linear regression on log ferritin concentration ( $\mu g / l)$

\begin{tabular}{lccrc}
\hline Variable & Adjusted regression coefficient & $95 \%$ CI & \multicolumn{1}{c}{ t } & \multicolumn{1}{c}{ p Value } \\
\hline Birth weight & $0.15^{\star}$ & 0.12 to 0.19 & 9.36 & $<0.0001$ \\
Weight at 8 months $(\mathrm{kg})$ & $-0.044 \dagger$ & -0.061 to -0.027 & $-5 \cdot 11$ & $<0.0001$ \\
Sex & $0.069 \ddagger$ & 0.036 to 0.101 & 4.15 & $<0.0001$
\end{tabular}

Sex

$R^{2}=0 \cdot 132, \mathrm{n}=751$.

«Equivalent to an increase in ferritin of $4 \%$ for a $1 \mathrm{~kg}$ increase in birth weight.

tEquivalent to a decrease in ferritin of $10 \%$ for a $1 \mathrm{~kg}$ increase in weight at 8 months.

fEquivalent to female children having ferritin concentrations $17 \%$ higher than male children.

specifically excluding individuals who are 'unhealthy'. It is possible that the results at the bottom end of our range could be derived from ill infants. However, this seems unlikely as the inclusion of individuals with abnormal conditions would result in a skewed normal range, while the distribution we have derived for haemoglobin is a symmetrical, normal one (fig 1).

These data challenge the traditional cut offs used for definition of anaemia at 8 months of age. Using a haemoglobin cut off of $110 \mathrm{~g} / \mathrm{l}^{3}$ or $107 \mathrm{~g} / 1,9$ over $20 \%$ of the apparently healthy normal population were identified as being anaemic - numbers clearly inappropriate for this age. Conversely, using the 5 th centile from the distribution generated by these data, a cut off of $96 \mathrm{~g} / \mathrm{l}$ for boys and $99 \mathrm{~g} / \mathrm{l}$ for girls would be produced. These may be more realistic criteria, using the capillary methodology available. Many clinics are now moving towards using capillary samples, and these new norms will be useful in identifying which children require further investigation. Nevertheless it would be naive to assume that the distribution derived from a reference population should dictate criteria for intervention. The threshold for intervention should be based on knowledge of harmful prognosis, and this has not yet been determined.

There is no doubt that prolonged and severe iron deficiency anaemia has consequences for psychomotor development. ${ }^{12}$ What is not certain is whether mild anaemia with normal iron stores, or a reduction in iron stores without anaemia, have any long term consequences. The optimal age to screen the population is also not clear, and the Children in Focus study will allow these questions to be addressed, as the 1100 children will be followed up until the age of 7 years. These data will then be helpful in informing clinicians as to appropriate concentrations for intervention, and should help facilitate a rational approach towards screening for iron deficiency anaemia in the UK population. ${ }^{13}$

The Avon Longitudinal Study of Pregnancy and Childhood (ALSPAC) is part of the WHO initiated multicentre survey, the European Longitudinal Study of Pregnancy and Childhood (ELSPAC). The Children in Focus substudy is, however, unique to ALSPAC. Funding for the whole ALSPAC survey is derived from a variety of sources including the Wellcome Trust, the Department of Health, the Ministry of Agriculture and Fisheries and Foods, and the US National Institutes of Health. Specific funds for this anaemia study have been contributed by the South and West Regional Health Authority, the Department of Health, Cow and Gate, and the Meat and Divartock Livestock Commission. We gratefully acknowledge help in planning and conducting this anaemia study from Dr J James, Dr A Oakhill, Mr D Oakes, Mr N Farron, and Ms L McGrath. We are extremely grateful to all our funders, to the midwives and other health professionals who made the survey possible, but most of all to the parents and children who have taken part. Further information about the ALSPAC study can be obtained from Professor Golding.

1 James J, Evans J, Male P, et al. Iron deficiency in inner city preschool children: development of a general practice screening programme. $\mathcal{F} R$ Coll Gen Pract 1988; 38: 250-2.

2 Aukett MA, Parks YA, Scott PH, Wharton BA. Treatment with iron increases weight gain and psychomotor development. Arch Dis Child 1986; 61: 849-57.

3 World Health Organisation. Nutritional anaemia. Geneva: WHO, 1972. (WHO Technical Report Series No 50.)

4 Oski FA. Iron deficiency in infancy and childhood. $N$ Engl $f$ Med 1993; 329: 190-3.

5 Burman D. Haemoglobin levels in normal infants aged 3 to 24 months and the effect of iron. Arch Dis Child 1972; 47: 24 month

6 Duggan MB, Steel G, Harbottle EZ, Nobel C. Iron status, energy intake, and nutritional status of healthy young Asian children. Arch Dis Child 1991; 66: 1386-9.

7 Mills AF. Surveillance for anaemia: risk factors in patterns of milk intake. Arch Dis Child 1990; 65: 428-31.

8 Stevens D. Epidemiology of hypochromic anaemia in young children. Arch Dis Child 1991; 66: 886-9.

9 Yip R, Johnson C, Dallman PR. Age-related changes in laboratory values used in the diagnosis of anaemia and iron deficiency. Am $\mathcal{f}$ Clin Nutr 1984; 39: 427-36.

10 Dallman PR, Reeves J. Laboratory diagnosis of iron deficiency. In: Stekel A, ed. Iron nutrition in infancy and childhood. New York: Raven Press, 1984: 25-6.

11 Liappis N, Mallmann R, Schlebusch H. Comparison of the ferritin and iron concentration in capillary and venous ferritin and iron concentration in capillary and venous blood samples of children adolescents

12 Walter T, De Andraca I, Chadud P, et al. Iron deficiency anaemia: adverse effects on infant psychomotor development. Pediatrics 1989; 84: 7-17.

13 Department of Health (COMA). Weaning and weaning diet. London: HMSO, 1994. 